CUBO A Mathematical Journal

Vol.12, Noㅡ, (53-75). June 2010

\title{
Generalized quadrangles and subconstituent algebra ${ }^{1}$
}

\author{
FERNANDO LEVSTEIN \\ FaMAF-CIEM,UNC, \\ Universidad Nacional de Córdoba \\ Medina Allende y Haya de la Torre. CP 5000 - Córdoba, Argentina \\ email: levstein@famaf.unc.edu.ar \\ and \\ Carolina Maldonado \\ Departamento de Matemática, Centro de Ciências Exatas e da Natureza, \\ Universidade Federal de Pernambuco \\ Av. Prof. Luiz Freire, s/n Cidade Universitária - Recife, Brasil \\ email: cmaldona@famaf.unc.edu.ar
}

\begin{abstract}
The point graph of a generalized quadrangle $G Q(s, t)$ is a strongly regular graph $\Gamma=$ $\operatorname{srg}(\nu, \kappa, \lambda, \mu)$ whose parameters depend on $s$ and $t$. By a detailed analysis of the adjacency matrix we compute the Terwilliger algebra of this kind of graphs (and denoted it by $\mathcal{T}$ ). We find that there are only two non-isomorphic Terwilliger algebras for all the generalized quadrangles. The two classes correspond to wether $s^{2}=t$ or not. We decompose the algebra into direct sum of simple ideals. Considering the action $\mathcal{T} \times \mathbb{C}^{X} \longrightarrow \mathbb{C}^{X}$ we find the decomposition into irreducible $\mathcal{T}$-submodules of $\mathbb{C}^{X}$ (where $X$ is the set of vertices of the $\Gamma$ ).
\end{abstract}

\section{RESUMEN}

El grafo de puntos de un cuadrángulo generalizado $G Q(s, t)$ es un grafo fuertemente regular $\Gamma=\operatorname{srg}(\nu, \kappa, \lambda, \mu)$ cuyos parámetros dependen de $s$ y $t$. Mediante un análisis detallado de

\footnotetext{
${ }^{1}$ This work supported by FACEPE, CCEN UFPE and CIEM-FaMAF UNC, CONICET.
} 
la matriz de adyacencia, calculamos el álgebra de Terwilliger ( $\mathcal{T}$-álgebra) de esta familia de grafos. Encontramos que para todos los cuadrángulos generalizados, existen solo dos tipos no isomorfos de $\mathcal{T}$-álgebras asociadas. Dichas clases dependen de si $s^{2}=t$ o no. Descomponemos el álgebra en suma directa de ideales simples. Considerando la acción $\mathcal{T} \times \mathbb{C}^{X} \longrightarrow \mathbb{C}^{X}$ encontramos la descomposición de $\mathbb{C}^{X}$ en $\mathcal{T}$-submódulos irreducibles. $(X$ es el conjunto de vértices de $\Gamma$ )

Key words and phrases: strongly regular graphs, generalized quadrangles, Terwilliger algebra.

AMS (MOS) Subj. Class.: 05E30

\section{Introduction}

The subconstituent algebra was first introduced by P. Terwilliger in his paper [13]. It was defined on a class of combinatorial objects known as association schemes (see also [2, 3]). It is a noncommutative, finite dimensional, semisimple $\mathbb{C}$ algebra. We will denote it by $\mathcal{T}$.

It has been studied for many examples such as $P$ - and $Q$ - polynomial association schemes [6], distance-regular graph that supports a spin model [7], group association schemes [4, 5], strongly regular graphs [17].

In [8] it was given an explicit description of the $\mathcal{T}$-algebra of the hypercube and more generally in [10] of a Hamming scheme $H(d ; q)$. The case of the Johnson schemes it was analyzed in [9].

In this paper we focus on the $\mathcal{T}$-algebra of a special family of strongly regular graphs, which are examples of association schemes: generalized quadrangles $G Q(s, t)$.

They are indeed a subfamily of partial geometries defined in [1]. A strongly regular graph is associated to them, so we can study the $\mathcal{T}$-algebra of such a family. We show that there are only two non-isomorphic $\mathcal{T}$-algebras for all the generalized quadrangles. The two classes correspond to whether $s^{2}=t$ or not. We obtain the dimension of $\mathcal{T}$ in both cases. This is in agreement with the result expected from [17] that gives dimensions of the $\mathcal{T}$-algebra attached to a strongly regular graph. The particular class of $G Q\left(s, s^{2}\right)$ has a combinatorial characterization given by J.A. Thas in [16].

With a detailed analysis of the adjacency matrix, we obtain restriction on the parameters $(s, t)$ (also given in 1.2.2 of [12]).

The paper is organized as follows: in section 2 we give the basic definitions and comment on some known basic results of algebraic combinatorics. In section 3 we analyze the blocks of the matrices in $\mathcal{T}$ and we give a basis of $\mathcal{T}$ in Proposition 3.21.

In section 4 we find the simple ideals of $\mathcal{T}$ (Propositions 4.3, 4.4) and in Theorem 4.5 we decompose $\mathcal{T}$ into direct sum of simple ideals.

Finally in section 5 we give the irreducible $\mathcal{T}$-submodules of the action

$$
\mathcal{T} \times \mathbb{C}^{X} \longrightarrow \mathbb{C}^{X}
$$

(where $X$ is the set of vertices of the $\Gamma$ ). 


\section{Definitions}

\subsection{Strongly regular graphs}

Definition 2.1. (see [11]) A strongly regular graph $\Gamma=\operatorname{srg}(\nu, \kappa, \lambda, \mu)$ is a graph with $\nu$ vertices that is regular of degree $\kappa$ and that has the following properties:

- for any two adjacent vertices $x, y$ there are exactly $\lambda$ vertices adjacent to $x$ and to $y$

- for any two nonadjacent vertices $x, y$ there are exactly $\mu$ vertices adjacent to $x$ and to $y$

\subsection{Generalized Quadrangles}

Definition 2.2. (see [1] , [12])

A generalized quadrangle $G Q(s, t)$ is a system of points and lines with an incidence relation satisfying the axioms (1) - (4) below. We will use standard geometric language. A point incident with a line is said to lie on the line and the line is said to pass through the point. If two lines are incident with the same point, we say that they intersect.

\section{Axioms}

1. for any two distinct points there is at most one line passing through them;

2. there are exactly $r=t+1$ lines passing for each point;

3. there are exactly $k=s+1$ points lying on each line;

4. if a point $p$ does not lie on the line $l$, then there is exactly one line passing through $p$ and intersecting $l$

If two points lie on a common line, we say that they are collinear and we write $x \sim y$.

The point graph of a generalized quadrangle is the graph with the points of the quadrangle as vertices, and edges $\{x, y\}$ such that $x \sim y$.

It is well known by $[1,12]$ that the point graph of a $G Q(k-1, r-1)$ is a (possibly trivial) $\Gamma=$ $\operatorname{srg}(\nu, \kappa, \lambda, \mu)$ with:

$$
\nu=k(1+(k-1)(r-1)), \kappa=r(k-1), \lambda=k-2, \mu=r
$$

\subsection{Bose-Mesner algebra}

Let $\Gamma=\operatorname{srg}(\nu, \kappa, \lambda, \mu)$ be a strongly regular graph, $X$ be the set of vertices and

$$
\partial: X \times X \rightarrow\{0,1,2\}
$$

be the path-length distance for $\Gamma$. Let $\operatorname{Mat}_{X}(\mathbb{C})$ denote the $\mathbb{C}$-algebra of matrices with complex entries, where the rows and columns are indexed by $X$. 
Definition 2.3. The adjacency matrix of $\Gamma$ of is the following $(0,1)$-matrix in $\operatorname{Mat}_{X}(\mathbb{C})$ :

$$
(A)_{x y}= \begin{cases}1 & \text { if } \partial(x, y)=1 \\ 0 & \text { otherwise }\end{cases}
$$

Proposition 2.4. (see [11])

Let $\Gamma=\operatorname{srg}(\nu, \kappa, \lambda, \mu)$ be a strongly regular graph, $A$ the adjacency matrix of $\Gamma$ and $I, J \in$ $\operatorname{Mat}_{X}(\mathbb{C})$ the identity and the full ones matrix respectively. Then

$$
\begin{aligned}
A J & =\kappa J \\
A^{2}+(\mu-\lambda) A+(\mu-\kappa) I & =\mu J
\end{aligned}
$$

Proof. By definitions 2.1 and 2.3; $A$ is a symmetric matrix with $\kappa$ 1's on each row and column. This proves equation (2). To prove (3) we observe that defining

$$
\left(A_{2}\right)_{x y}=\left\{\begin{array}{ll}
1 & \text { if } \partial(x, y)=2 \\
0 & \text { otherwise }
\end{array},\right.
$$

axioms of definition 2.1 imply that

$$
I+A+A_{2}=J
$$

$\left(A_{2} \neq J-I\right)$ otherwise $\Gamma$ would be a complete graph).

Computing:

$$
\begin{aligned}
\left(A^{2}\right)_{x y} & =\Sigma_{z \in X} A_{x z} A_{z y} \\
& =\mid\{z: \partial(x, z)=1 \text { and } \partial(z, y)=1\} \mid \\
& =\left\{\begin{array}{rlr}
\kappa & \text { if } \quad x=y \\
\lambda & \text { if } & \partial(x, y)=1 \\
\mu & \text { if } & \partial(x, y)=2
\end{array}\right.
\end{aligned}
$$

Therefore

$$
\begin{aligned}
A^{2} & =\kappa I+\lambda A+\mu A_{2} \\
& =\kappa I+\lambda A+\mu(J-I-A)
\end{aligned}
$$

which implies the (3).

Definition 2.5. (see [2], [3] )

The Bose-Mesner algebra of a strongly regular graph $\Gamma$ is the 3-dimensional algebra of matrices in $\operatorname{Mat}_{X}(\mathbb{C})$ which are linear combinations of $I, J$ and $A$. We denoted it by $\mathcal{A}$.

That this is indeed an algebra is a consequence of equations (2) and (3) in Proposition 2.4.

The following facts are well known in algebraic combinatorics (see $[2,3])$. 
The algebra $\mathcal{A}$ consists of symmetric commuting matrices and identifying

$$
\mathbb{C}^{X}=\{f: X \rightarrow \mathbb{C}\}
$$

we can consider for all $M \in \mathcal{A}$ the action:

$$
M \times \mathbb{C}^{X} \rightarrow \mathbb{C}^{X} .
$$

Since $\{I, J, A\}$ consists of symmetric commuting matrices, they are diagonalyzed simultaneously by a unitary matrix. That is, we have a decomposition of $\mathbb{C}^{X}$ into common eingenspaces of $I, J, A$. The number of eigenspaces is $2+1$ since any strongly regular graph has diameter $=2$ (diameter:= the greatest distance in the graph).

Therefore, let $\Gamma$ be a strongly regular graph,

$$
\mathbb{C}^{X}=V_{0} \oplus V_{1} \oplus V_{2}
$$

be such a decomposition and let $E_{i}, i=0,1,2$ be the orthogonal projections

$$
E_{i}: \mathbb{C}^{X} \rightarrow V_{i}
$$

expressed in matrix form with respect to the canonical basis $\left\{e_{i}\right\} i=1 \ldots|X|$. Then,

$$
\begin{aligned}
E_{0} & =\frac{1}{|X|} J(J \text { the matrix of all } 1, \mathrm{~s}) \\
E_{0}+E_{1}+E_{2} & =I \\
E_{i} E_{j} & =\delta_{i j} E_{i}
\end{aligned}
$$

The $E_{i}$ are called the primitive idempotents of $\Gamma$.

\subsection{Dual Bose-Mesner algebra}

Definition 2.6. (see [13]) The $i^{\text {th }}$ dual idempotent with respect to the vertex $x$ denoted by $E_{i}^{*}:=E_{i}^{*}(x)$ is the diagonal matrix in $M a t_{X}(\mathbb{C})$ defined by

$$
\left(E_{i}^{*}\right)_{y y}= \begin{cases}1 & \text { if } \partial(x, y)=i \\ 0 & \text { if } \partial(x, y) \neq i\end{cases}
$$

Lemma 2.7. The matrices $\left\{E_{i}^{*}\right\}_{i=0}^{2}$ satisfy the following equations:

$$
\begin{aligned}
E_{0}^{*}+E_{1}^{*}+E_{2}^{*} & =I \\
E_{i}^{* t} & =E_{i}^{*} \\
E_{i}^{*} E_{j}^{*} & =\delta_{i j} E_{i}^{*}
\end{aligned}
$$

Proof. Its follows straightforward from definition above.

Definition 2.8. Let $\Gamma$ be a strongly regular graph. For $x \in X$, the Dual Bose-Mesner algebra of $\Gamma$ with respect to $x$, is the 3-dimensional algebra of matrices in $M a t_{X}(\mathbb{C})$ which are linear combinations of $\left\{E_{i}^{*}\right\}_{i=0}^{2}$. We denoted it by $\mathcal{A}^{*}:=\mathcal{A}^{*}(x)$. 
That this is indeed an algebra is a consequence of equations (4),(5) and (6) in the previous Lemma.

\subsection{Terwilliger algebra}

Definition 2.9. (see [13]) Let $\Gamma$ be a strongly regular graph and $X$ be its set of vertices. The subconstituent or Terwilliger algebra of $\Gamma$ with respect to the vertex $x \in X$ is the algebra generated by the Bose-Mesner algebra $\mathcal{A}:=\mathcal{A}(x)$ and the dual Bose-Mesner algebra $\mathcal{A}^{*}:=\mathcal{A}^{*}(x)$. We denote this algebra by $\mathcal{T}:=\mathcal{T}(x)$.

Remark 2.10. $\mathcal{T}$ is closed under the conjugate-transpose map, so it is semi-simple.

\section{$3 \quad \mathcal{T}$-algebra of $G Q(k-1, r-1)$.}

In this section we consider a connected strongly regular graph $\Gamma=\operatorname{srg}(\nu, \kappa, \lambda, \mu)$ coming from a generalized quadrangle $G Q(k-1, r-1)$.

We fix $x_{0} \in X$ and we analyze the associated $\mathcal{T}\left(x_{0}\right)$-algebra .

In the following we analyze the structure of the matrices belonging to $\mathcal{T}$ in a more detailed way .

Lemma 3.1. For all $T \in \mathcal{T}, T$ is generated by $A, E_{0}^{*}, E_{1}^{*}, E_{2}^{*}$

Proof. By definition $\mathcal{T}$ is generated by the algebras $\mathcal{A}=\langle\{I, J, A\}\rangle$ and $\mathcal{A}^{*}=\left\langle\left\{E_{0}^{*}, E_{1}^{*}, E_{2}^{*}\right\}\right\rangle$. That is $\mathcal{T}$ consist on sum and products of matrices in $\left\{I, J, A, E_{0}^{*}, E_{1}^{*}, E_{2}^{*}\right\}$.

Equation (3) shows that $J$ can be obtained as a linear combination of $A^{2}, A, I$ and equation (4) shows that the identity is the sum of $\left\{E_{i}^{*}\right\}_{i=0}^{2}$.

Remark 3.2. It is well known that for the point graph of a generalized quadrangle the isomorphism class of $\mathcal{T}(x)$ is independent on the vertex $x$, since the group of automorphism of the graph $\Gamma$ acts transitively on $X$ preserving the distance.

Then any automorphism

$$
\begin{array}{clcc}
g: X & \rightarrow & X & \\
x & \rightarrow & y, & \text { induces an isomorphism } \\
T_{g}: \mathcal{T}(x) & \rightarrow & \mathcal{T}(y) . & \\
M^{x} & \rightarrow & M^{y} & \\
\text { where } M_{u v}^{y} & := & M_{g^{-1} u g^{-1}}^{x}, & \text { for } M^{x} \in \mathcal{T}(x), M^{y} \in \mathcal{T}(y) ; u, v \in X \\
\text { and then } \mathcal{T}(x) & \simeq & \mathcal{T}(y) &
\end{array}
$$

In view of Lemma 3.1 we consider the products

$$
E_{i}^{*} A E_{j}^{*} \quad i, j=0,1,2
$$

where $A$ is the adjacency matrix and $E_{i}^{*}$ the dual idempotents of definitions 2.3 and 2.6 respectively. 


\subsection{Block analysis}

We will use an order of the set of vertices $X$ that allows us to analyze the matrices in $\mathcal{T}\left(x_{0}\right)$ in a convenient way.

Let $x_{0}$ be a fixed vertex of $X$. Take

$$
\Omega_{0}=\left\{x_{0}\right\}, \Omega_{i}=\left\{y \in X \mid \partial\left(x_{0}, y\right)=i\right\}
$$

We consider the matrices in $\operatorname{Mat}_{X}(\mathbb{C})$ indexed by the blocks $\Omega_{i} \times \Omega_{j}$.

Just to give examples, we have:

$\left.E_{0}^{*}=\begin{array}{c|c|c|c}x_{0} & x_{0} & \Omega_{2} \\ \hline \Omega_{1} & 0 & 0 & 0 \\ \hline \Omega_{2} & 0 & 0 & 0\end{array}\right]$

$\left.E_{1}^{*}=\begin{array}{c|c|c|c}x_{0} & 0 & x_{0} & \Omega_{2} \\ \hline \Omega_{1} & 0 & I & 0 \\ \hline \Omega_{2} & 0 & 0 & 0\end{array}\right]$

$\left.E_{1}^{*} A E_{2}^{*}=\begin{array}{c|c|c|c}x_{0} & \Omega_{1} & \Omega_{2} \\ \hline \Omega_{1} & 0 & 0 & 0 \\ \hline \Omega_{2} & 0 & 0 & A_{\mid \Omega_{1} \times \Omega_{2}} \\ \hline & & 0\end{array}\right]$

We will denote

$$
P:=A_{\mid \Omega_{1} \times \Omega_{1}} Q:=A_{\mid \Omega_{1} \times \Omega_{2}} S:=A_{\mid \Omega_{2} \times \Omega_{2}}
$$

and $I_{i k}:=I_{\mid \Omega_{i} \times \Omega_{k}}, J_{i k}:=J_{\mid \Omega_{i} \times \Omega_{k}}$, that is the submatrix of $I$ or $J$ of size $\Omega_{i} \times \Omega_{k}$.

Then $A_{\mid x_{0} \times \Omega_{1}}=J_{01}=(1, \ldots, 1)$ and since $A$ is symmetric we have

$$
A_{\mid \Omega_{2} \times \Omega_{1}}=Q^{t}, A_{\mid \Omega_{1} \times x_{0}}=J_{01}^{t}=(1, \ldots, 1)^{t} .
$$

Then $A$ looks like: 


\begin{tabular}{|c|c|c|c|c|}
\hline & $x_{0}$ & $x_{0}$ & $\Omega_{1}$ & $\begin{array}{c}\Omega_{2} \\
0\end{array}$ \\
\hline$A=$ & $\Omega_{1}$ & $\begin{array}{l}\vdots \\
1\end{array}$ & $P$ & $Q$ \\
\hline & $\Omega_{2}$ & 0 & $Q^{t}$ & $S$ \\
\hline
\end{tabular}

The following lemma gives some descriptions of blocks of $A$.

\section{Lemma 3.3.}

Let $\Gamma=\operatorname{srg}(\nu, \kappa, \lambda, \mu)$ be a srg associated to a generalized quadrangle $G Q(k-1, r-1)$ (that is the parameters $(\nu, \kappa, \lambda, \mu)$ satisfy equations in (1)). Let $J_{k l}, P, Q, S$ be defined as above. Then

1. $A_{\mid x_{0} \times \Omega_{1}}=J_{01}$

2. $J_{10}=J_{01}^{t}$

3. $\left|\Omega_{1}\right|=r(k-1) ;\left|\Omega_{2}\right|=(r-1)(k-1)^{2}$

4. $P$ is a block of size $\left|\Omega_{1}\right| \times\left|\Omega_{1}\right|$ with $(k-2) 1^{\prime}$ s on each row and column,

5. $Q$ is a block of size $\left|\Omega_{1}\right| \times\left|\Omega_{2}\right|$ with $(r-1)(k-1) 1^{\prime}$ s on each row and $r 1^{\prime}$ s on each column and

6. $S$ has size $\left|\Omega_{2}\right| \times\left|\Omega_{2}\right|$ with $r(k-2) 1^{\prime}$ s on each row and column.

Proof.

- (1) holds since by definition of $A$, the block indexed by $x_{0} \times \Omega_{1}$ is the set of neighbors of $x_{0}$.

- (2) holds since $A$ is symmetric.

- (3) holds since $\left|\Omega_{1}\right|=\kappa$ (the degree of $\Gamma$ ) and $1+\left|\Omega_{1}\right|+\left|\Omega_{2}\right|=\nu$ (the number of vertices of $\Gamma$ ). Parameters $\kappa, \nu$ are given in Equations (1).

- Assertion (4) holds since for a fixed $x \in \Omega_{1}$ there are $\lambda=k-2$ neighbors of $x$ in $\Omega_{1}$.

- On the same way for a fixed $x \in \Omega_{2}$ there are $\mu=r$ neighbors of $x$ in $\Omega_{1}$ which implies that $Q$ has $r$ 1's on each column. The number of 1's on each row of $Q$ is $\left|\Omega_{1}\right|-(k-2)-1$.

- The number of 1's on each row and column of $S$ is $\left|\Omega_{1}\right|-r$. 
Remark 3.4. We have already discussed that in order to describe $\mathcal{T}$ we should analyze the products among the matrices in $\left\{E_{i}^{*} A E_{j}^{*}\right\}_{i, j=0,1,2}$. That is essentially the products among the blocks $J_{01}, J_{10}, P, Q, Q^{t}$ and $S$.

In the following subsections we analyze the structure of each block $\Omega_{i} \times \Omega_{j}$ and finally we give a basis for each one.

\section{$3.2 \Omega_{1} \times \Omega_{1}$-block}

We start giving expressions for some products belonging to the $\Omega_{1} \times \Omega_{1}$-block: $\left\{P^{n}, Q Q^{t}, P J_{11}, J_{11} P, J_{10} J_{01}\right\}$.

We describe the powers of $P$.

Lemma 3.5. $P$ satisfies $P^{2}=(k-3) P+(k-2) I_{11}$

Proof. The $\Omega_{1} \times \Omega_{1}$-block has size $r(k-1) \times r(k-1)$ and $P$ has $(k-2) 1$ 's on each row and column. It is indexed by the vertices in $\Omega_{1}$.

It has a one in the $\left(x_{i}, x_{j}\right)$ entry if and only if the common neighbors $x_{i}, x_{j}$ of $x_{0}$ form an edge of the graph $\Gamma$.

As the equation for $P$ does not depend on the order of the vertices of $\Omega_{1}$ we will consider a special ordering in which $P$ has a simple form.

We label the vertices in the following way: $\Omega_{0}=\left\{x_{0}\right\}$ and $l_{1}, l_{2} \ldots l_{r}$ the $r$ lines passing through the point $x_{0}$. We call $x_{1,1}, x_{1,2}, \ldots x_{1, k-1}$ the $(k-1)$ points lying on the $l_{1} \backslash\left\{x_{0}\right\} ; x_{2,1}, x_{2,2}, \ldots x_{2, k-1}$ the points lying on the $l_{2} \backslash\left\{x_{0}\right\}$ and so on.

All the points lying on the same line are collinear points. Then any two of them form an edge on the point graph of the generalized quadrangle. If we order the vertices of the $\Omega_{1} \times \Omega_{1}$-block with the order of the lines, that is

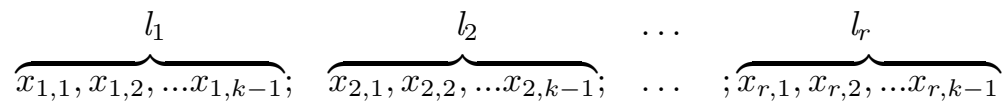

$\mathrm{P}$ has the form:

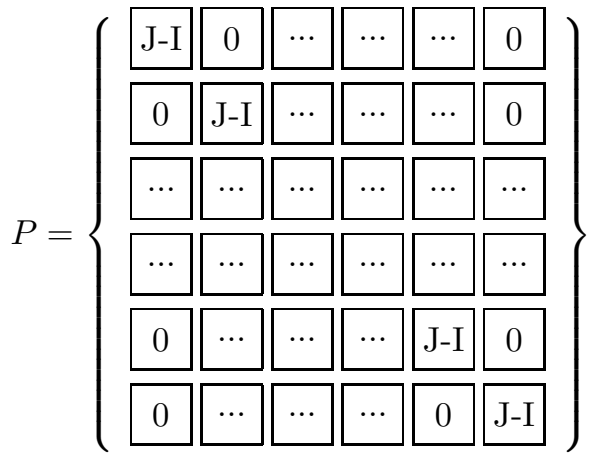

and is not difficult to see that $P^{2}=(k-3) P+(k-2) I_{11}$, which implies the lemma. 
Corollary 3.6. The matrices $P, I_{11}$ and $J_{11}$ are independent and $P^{2}$ depends on $P$ and $I_{11}$.

Proof. $P, I_{11}$ and $J_{11}$ are independent, otherwise the relation among them should be $P=J_{11}-I_{11}$. But this would imply that the graph is not connected. Since we omit these cases we have the conclusion.

Lemma 3.7. Using the same ordering as above for $\Omega_{1}$ and any order for $\Omega_{2}$ we have

$$
\begin{aligned}
Q Q^{t} & =(r-1)(k-2) I_{11}-(r-1) P+(r-1) J_{11} \\
J_{10} J_{01} & =r(k-1) J_{11} \\
P J_{11} & =(k-2) J_{11}
\end{aligned}
$$

Proof. Equating the $\Omega_{1} \times \Omega_{1}$-block of (3) we have

$$
J_{10} J_{01}+P^{2}+Q Q^{t}+(\mu-\lambda) P+(\mu-\kappa) I_{11}=\mu J_{11}
$$

Replacing the parameters $\lambda, \mu, \kappa$ by Equation (1) and $P^{2}$ as in the previous lemma, we get

$$
J_{11}+(k-3) P+(k-2) I_{11}+Q Q^{t}+(r-k+2) P-r(k-2) I_{11}=r J_{11}
$$

which implies the expression for $Q Q^{t}$. The other equations are easy to check.

Proposition 3.8. The products $P^{n}, Q Q^{t}, J_{10} J_{01}$ y $P J_{11}$ can be expressed as a linear combinations of $P, I_{11}, J_{11}$ and they are linearly independent.

Proof. It follows directly from lemmas 3.5 and 3.7 .

\section{3 $\Omega_{1} \times \Omega_{2}$ - block}

Now we give expressions for the products $P Q, Q S, J_{11} Q, Q J_{22}, J_{12} S$

Lemma 3.9. Using the same ordering for $\Omega_{1}$ as in the Lemma 3.5 the following equation holds:

$$
P Q=J_{12}-Q
$$

Proof. The $\Omega_{1} \times \Omega_{2}$-block has size $r(k-1) \times(r-1)(k-1)^{2}$. From Lemma 3.3, we now that $Q$ has $(r-1)(k-1) 1$ 's on each row and $r$ 's on each column. By hypothesis, the rows of $Q$ are indexed by the vertices of the lines $l_{1}, l_{2}, \ldots l_{r}$.

The columns are indexed by the set $\Omega_{2}$ (the vertices which are not neighbors of $x_{0}$ ).

Let $\left(x_{i j}, y\right)$ be an entry of the product $P Q$ where $y \in \Omega_{2}$ and $x_{i j}$ is the $i^{t h}$ vertex of the line $l_{j}$. Then

$$
(P Q)_{\left(x_{i j}, y\right)}=\sum_{m=1}^{r} \sum_{n=1}^{k-1} P_{\left(x_{i j}, x_{m n}\right)} Q_{\left(x_{m n}, y\right)} .
$$

Since $P$ vanishes on the vertices lying on different lines $\left(P_{\left(x_{i j}, x_{k l}\right)}=0\right.$ for $\left.i \neq k\right)$,

$$
(P Q)_{\left(x_{i j}, y\right)}=\sum_{n=1}^{k-1} P_{\left(x_{i j}, x_{i n}\right)} Q_{\left(x_{i n}, y\right)}
$$


Each vertex of $\Omega_{2}$ has exactly one neighbor on the line $l_{i}$ (fourth axiom of definition 2.2). Therefore for $y \in \Omega_{2}$ there exist a unique $x_{i n_{y}} \in l_{i}$ such that

$$
\left.Q_{(} x_{i j}, y\right)=\left\{\begin{array}{lll}
1 & \text { if } & j=n_{y} \\
0 & \text { if } & j \neq n_{y}
\end{array}\right.
$$

Then

$$
\begin{aligned}
(P Q)_{\left(x_{i j}, y\right)} & =\sum_{n=1}^{k-1} P_{\left(x_{i j}, x_{i n}\right)} Q_{\left(x_{i n}, y\right)} \\
& =P_{\left(x_{i j}, x_{i n y}\right)} \\
& =(J-I)_{\left(x_{i j}, x_{i n_{y}}\right)} \\
& =\left\{\begin{array}{rrr}
0 & \text { if } & j=n_{y} \\
1 & \text { if } & j \neq n_{y}
\end{array}\right. \\
& =(J-Q)_{\left(x_{i j}, x_{i n_{y}}\right)},
\end{aligned}
$$

which proves the lemma.

Lemma 3.10. $Q$ and $S$ satisfy:

$$
\begin{aligned}
Q S & =(r-1) J_{12}+(k-1-r) Q, & J_{11} Q & =r J_{12}, \\
Q J_{22} & =(r-1)(k-1) J_{12}, & J_{12} S & =r(k-2) J_{12}
\end{aligned}
$$

Proof. The $\Omega_{1} \times \Omega_{2}$-block of identity (3) for $A$ gives $P Q+Q S+(r-k+2) Q=r J_{12}$.

Replacing $P Q$ by the result of the lemma 3.9 we have the first equation. For the other equations, we use that $Q$ has $(r-1)(k-1) 1^{\prime} s$ on each row and $r 1^{\prime} s$ on each column, and $S$ has $r(k-2) 1^{\prime} s$ on each row and column.

Proposition 3.11. The products $P^{n} Q, S^{n} Q, J_{11} Q, Q J_{22}, J_{12} S$ can be expressed as linear combinations of $Q$ and $J_{12}$.

Proof. Using lemmas 3.5 and 3.9 we can prove inductively that $P^{n} Q$ is a linear combination of $Q$ and $J_{12}$. On the same way Lemma 3.10 proves inductively the assertion for $S^{n} Q$. The other equations were also proved in Lemma 3.10.

\section{$3.4 \Omega_{2} \times \Omega_{2}$-block}

In the following, we give an expression for $S^{n}, Q^{t} Q$ and $J_{22} S$.

Lemma 3.12.

$$
Q^{t} Q=-S^{2}+r(k-2) I_{22}+(k-2-r) S+r J_{22}, \quad S J_{22}=r(k-2) J_{22}
$$

Proof. The $\Omega_{2} \times \Omega_{2}$-block of identity (3) for $A$ gives the first equation. The matrix $S$ has $r(k-2) 1^{\prime} s$ on each row and column thus we get the second equation. 


\section{Proposition 3.13.}

$$
\begin{aligned}
S^{3} & =((k-1-r)+(k-2-r)) S^{2}+(r(k-2)-(k-1-r)(k-2-r)) S \\
& -((k-1-r)+r(k-2)) I_{22}+(r(r-1)(k-2)) J_{22} .
\end{aligned}
$$

Equivalently if we denote

$$
\lambda_{1}=k-r-1, \lambda_{2}=k-2, \lambda_{3}=-r,
$$

then $S$ satisfies the equation

$$
\left(S-\lambda_{1} I_{22}\right)\left(S-\lambda_{2} I_{22}\right)\left(S-\lambda_{3} I_{22}\right)=r(r-1) J_{22}
$$

Proof. Postmultiplying $Q^{t} Q$ given in (3.12) by $S$ we have

$$
Q^{t} Q S=-S^{3}+r(k-2) S+(k-2-r) S^{2}+r^{2}(k-2) J_{22}
$$

Replacing $Q S$ by the expression given in the lemma 3.10

$$
\begin{aligned}
& Q^{t}\left((k-1-r) Q+(r-1) J_{22}\right)=-S^{3}+r(k-2) S+(k-2-r) S^{2}+r^{2}(k-2) J_{22}, \\
& S^{3}=-(k-1-r) Q^{t} Q-r(r-1) J_{22}+r(k-2) S+(k-2-r) S^{2}+r^{2}(k-2) J_{22} .
\end{aligned}
$$

Replacing $Q^{t} Q$ by 3.12 we have the first equation, that is equivalent to

$$
\begin{array}{ll}
S^{3} & -((k-1-r)+(k-2-r)) S^{2}-(r(k-2)-(k-1-r)(k-2-r)) S \\
+ & ((k-1-r)+r(k-2)) I_{22}=(r(r-1)(k-2)) J_{22}
\end{array}
$$

At this moment we can not tell whether $S^{2}, S, I_{22}$ and $J_{22}$ are independent or not.

In what follows we are going to show that $S^{2}$ depends on $S I_{22}$ and $J_{22}$ if and only if the parameters of the generalized quadrangle satisfy $(k-1)^{2}=r-1$.

Corollary 3.14. Denoting

$$
\begin{array}{lll}
\lambda_{0}=r(k-2), & \lambda_{1}=k-r-1, \\
\lambda_{2}=k-2, & \lambda_{3}=-r
\end{array}
$$

$S$ satisfies the equation $\left(S-\lambda_{0} I_{22}\right)\left(S-\lambda_{1} I_{22}\right)\left(S-\lambda_{2} I_{22}\right)\left(S-\lambda_{3} I_{22}\right)=0$

Proof. By Lemma The $\Omega_{2} \times \Omega_{2}$-block has size $(r-1)(k-1)^{2} \times(r-1)(k-1)^{2} . S$ has $r(k-2) 1$ 's on each row and on each column. So we have $S J_{22}=r(k-2) J_{22}$. Thus, if we multiply (8) by $S-r(k-2) I_{22}$ we have the corollary. 
This corollary implies that $S$ has at most four different eigenvalues. We know that $r(k-2)$ is an eigenvalue associated to the one dimensional eigenspace generated by $(1,1, \ldots, 1)$. then by PerronFrobenious Theorem it has multiplicity one.

Let $d_{i}=\operatorname{dim} V_{\lambda_{i}}$, where $V_{\lambda_{i}}$ is the eigenspace corresponding to $\lambda_{i}$. We have the following linear system of equations on $d_{0}$ and the unknowns: $\left\{d_{i}\right\}_{i=1}^{3}$

$$
\begin{aligned}
& \operatorname{tr} I=\quad \sum_{i=0}^{3} d_{i} \quad=(r-1)(k-1)^{2}, \\
& \operatorname{tr} S=\quad \sum_{i=0}^{3} \lambda_{i} d_{i}=0 \\
& \text { and } \operatorname{tr} S^{2}=\quad \sum_{i=0}^{3} \lambda_{i}{ }^{2} d_{i}=r(k-2)(r-1)(k-1)^{2} \text {, }
\end{aligned}
$$

then

$$
\begin{aligned}
& \operatorname{tr} I=\quad \sum_{i=1}^{3} d_{i}=(r-1)(k-1)^{2}-1, \\
& \operatorname{tr} S=\quad \sum_{i=1}^{3} \lambda_{i} d_{i}=-r(k-2) \\
& \text { and } \operatorname{tr} S^{2}=\quad \sum_{i=1}^{3} \lambda_{i}{ }^{2} d_{i} \quad=r(k-2)(r-1)(k-1)^{2}-(r(k-2))^{2} \text {, }
\end{aligned}
$$

with set of solutions

$$
\begin{array}{rcc}
d_{1} & = & r(k-2) \\
d_{2} & = & \frac{r(k-1)^{2}(r-2)}{(k+r-2)} \\
\text { and } d_{3} & = & \frac{(k-2)(r-1)\left((k-1)^{2}-(r-1)\right)}{(k+r-2)} .
\end{array}
$$

As the dimensions are non negative integers we have $(k-1)^{2} \geq(r-1)$, which is known as the inequality of D.G. Higman.(page 3 of [12])

In general $k+r-2$ must divide both $(k-2)(r-1)\left((k-1)^{2}-(r-1)\right)$ and $r(k-1)^{2}(r-2)$ if the parameters correspond to a generalized quadrangle.

Dimensions $\left\{d_{i}\right\}_{i=1}^{3}$ are always positive integers unless $(k-1)^{2}=r-1$, in which case $d_{3}=0$ and $\lambda_{3}$ is not an eigenvalue. Thus we have the following:

Proposition 3.15. $S$ has $\lambda_{3}=-r$ as eigenvalue if and only if the parameters $r$ and $k$ satisfy $(k-1)^{2}>r-1$.

Proof. It follows by the comments above.

Corollary 3.16. The matrices $S, I_{22}, J_{22}$ are linearly independent. $S^{2}$ depends on such matrices if and only if $(k-1)^{2}=r-1$

Proof. We have seen in Proposition 3.13 that the vector space generated by $\left\{S^{n}\right\}_{n \geq 0}$ has dimension 3 or 4. This depends on the minimal polynomial of $S$ and we have shown it has 3 different eigenvalues if and only if $(k-1)^{2}=r-1$.

Proposition 3.17. The products $\left\{Q^{t} Q, J_{22} S,\left\{S^{n}\right\}_{n \geq 0}\right\}$ can be expressed as a linear combinations of $S, I_{22}$ and $J_{22}$, if and only if the parameters $r, k$ of the generalized quadrangle satisfy $(k-1)^{2}=r-1$. Otherwise $S^{2}, S, I_{22}$ and $J_{22}$ span these products. 
Proof. Follows directly from Lemma 3.10 and Corollary 3.16 .

Theorem 3.18. The following spanning set are basis for the corresponding blocks.

$$
\begin{aligned}
\left\{x_{0}\right\} \times \Omega_{i} & =\left\langle J_{0 i}\right\rangle \quad i=0,1,2 \\
\Omega_{1} \times \Omega_{1} & =\left\langle\left\{I_{11}, J_{11}, P\right\}\right\rangle \\
\Omega_{1} \times \Omega_{2} & =\left\langle\left\{J_{12}, Q\right\}\right\rangle \\
\Omega_{2} \times \Omega_{2} & =\left\langle\left\{I_{22}, J_{22}, S\right\}\right\rangle \Leftrightarrow(k-1)^{2}=r-1 \\
& =\left\langle\left\{I_{22}, J_{22}, S, S^{2}\right\}\right\rangle \Leftrightarrow(k-1)^{2} \neq r-1
\end{aligned}
$$

Proof. It follows straightforward from Propositions 3.8, 3.11 and 3.17.

\subsection{Basis for $\mathcal{T}$ as a vector space}

The previous block-analysis allows to give a basis (as a vector space) of the $\mathcal{T}$-algebra attached to a $G Q(k-1, r-1)$. Actually we have analyzed the blocks of arbitrary matrices in $\mathcal{T}$. To be rigorous we should embed each block in $\operatorname{Mat}_{X}(\mathbb{C})$. To do this we propose the following

Definition 3.19. Let $B$ an arbitrary block indexed by the vertices in $\left\{\Omega_{i} \times \Omega_{j}\right\} i, j=0, \ldots 2$. We identify the block $B$ with a matrix $\iota(B)$ in $M a_{X}(\mathbb{C})$ in the following way:

$$
\iota(B)_{x y}=\left\{\begin{array}{ccc}
B_{x y} & i f(x, y) & \Omega_{i} \times \Omega_{j} \\
0 & \text { otherwise }
\end{array}\right.
$$

Example 3.20. Let $B$ be a block-matrix indexed by $\Omega_{2} \times \Omega_{1}$. Then

$\left.\iota(B)=\begin{array}{c|c|c|c}x_{0} & \Omega_{1} & \Omega_{2} \\ \hline x_{0} & 0 & 0 & 0 \\ \hline \Omega_{1} & 0 & 0 & 0 \\ \hline \Omega_{2} & 0 & B & 0\end{array}\right]$

Proposition 3.21. If the parameters of $G Q(k-1, r-1)$ satisfy $(k-1)^{2} \neq r-1$ then

$$
\begin{aligned}
& \mathcal{T}=\left\langle\left\{\left\{\iota\left(J_{i j}\right)\right\}_{i, j=0}^{2},\left\{\iota\left(I_{j j}\right)\right\}_{j=1}^{2}, \iota(P), \iota(Q), \iota\left(Q^{t}\right), \iota(S), \iota\left(S^{2}\right)\right\}\right\rangle \text { otherwise } \\
& \mathcal{T}=\left\langle\left\{\left\{\iota\left(J_{i j}\right)\right\}_{i, j=0}^{2},\left\{\iota\left(I_{j j}\right)\right\}_{j=1}^{2}, \iota(P), \iota(Q), \iota\left(Q^{t}\right), \iota(S)\right\}\right\rangle .
\end{aligned}
$$

Therefore $\quad \operatorname{dim}(\mathcal{T})=16$ or $\operatorname{dim}(\mathcal{T})=15$ respectively.

Proof. By Theorem 3.18 , the matrices

$$
\left\{\iota\left(J_{m n}\right)\right\}_{m, n=0}^{2},\left\{\iota\left(I_{m m}\right)\right\}_{m=1}^{2}, \iota(P), \iota(Q), \iota\left(Q^{t}\right), \iota(S)
$$


and eventually $\iota\left(S^{2}\right)$ (when $(k-1)^{2} \neq r-1$ ) give a basis (as a vector space) of a subalgebra of $\mathcal{T}$. This subalgebra contains the adjacency matrix $A$ and the dual idempotents $\left\{E_{i}^{*}\right\}$ since

$$
\begin{aligned}
A & =\iota\left(J_{00}\right)+\iota\left(J_{10}\right)+\iota\left(J_{10}\right)^{t}+\iota(P)+\iota(Q)+\iota\left(Q^{t}\right)+\iota(S) \\
E_{m}^{*} & =\iota\left(I_{m m}\right) .
\end{aligned}
$$

Therefore it coincides with $\mathcal{T}$.

\section{Simple ideals of $\mathcal{T}$}

In this section we decompose $\mathcal{T}$ as a direct sum of orthogonal simple ideals. We will guide us by the expression given by Proposition 3.21. There is one ideal present in every $\mathcal{T}$-algebra: the ideal $\mathcal{M}$ linearly generated by $\left\{\iota\left(J_{m n}\right)\right\}_{m, n=0}^{2}$.

Definition 4.1. For $m, n=0,1,2$ let $M_{m n} \in M_{a t}(\mathbb{C})$ be:

$$
M_{m n}=\frac{1}{\sqrt{\left|\Omega_{m}\right|\left|\Omega_{n}\right|}} \iota\left(J_{m n}\right)
$$

Proposition 4.2. The vector subspace $\mathcal{M}=\left\langle\left\{M_{m n}\right\}_{m, n=0}^{2}\right\rangle$ is a simple ideal of $\mathcal{T}$ and $\mathcal{M} \simeq$ $\operatorname{End}\left(\mathbb{C}^{3}\right)$.

Proof. It not difficult to prove that

$$
M_{m n} M_{p q}=\delta_{n p} M_{m q} \quad m, n, p, q=0,1,2
$$

which implies the proposition.

Using standard techniques we compute the following basis for the second ideal.

Let us denote

$$
\begin{array}{lll}
N_{11}=\frac{1}{k-1} \iota\left((k-2) I_{11}-P\right), & N_{12}=\frac{1}{(k-1) \sqrt{(k-1)(r-1)}} \iota\left((k-1) Q-J_{12}\right), \\
N_{21}=N_{12}^{t}, & N_{22}=\frac{1}{(k-1)^{2}(r-1)} \iota\left((k-1) Q^{t} Q-r J_{22}\right)
\end{array}
$$

We have the following

Proposition 4.3. The vector subspace $\mathcal{N}=\left\langle\left\{N_{m n}\right\}_{m, n=1}^{2}\right\rangle$ is a simple ideal of $\mathcal{T}$ orthogonal to the ideal $\mathcal{M}$ and $\mathcal{N} \simeq \operatorname{End}\left(\mathbb{C}^{2}\right)$.

Proof. It not difficult to prove that

$$
\begin{aligned}
N_{m n} N_{p q} & =\delta_{n p} N_{m q} & & m, n, p, q=1,2 \\
M N & =0 & & \forall M \in \mathcal{M}, N \in \mathcal{N},
\end{aligned}
$$

which implies the proposition. 
Now we give the expressions for the remaining one-dimensional ideals of $\mathcal{T}$. One can easily prove the following:

Proposition 4.4. The matrices

$$
\begin{aligned}
& P_{11}=\frac{1}{k-1} \iota\left(P+I_{11}-\frac{1}{r} J_{11}\right) \\
& R_{22}=\frac{1}{(r-1)(k-2+r)} \iota\left(S^{2}-(k-1-2 r) S-r(k-1-r) I_{22}-r J_{22}\right) \\
& S_{22}=\frac{1}{(k-1)(k-2+r)} \iota\left(S^{2}-(2 k-r-3) S+(k-1-r)(k-2) I_{22}-\frac{(k-2)(r-1)}{(k-1)} J_{22}\right)
\end{aligned}
$$

are idempotents and orthogonal to the ideals $\mathcal{M}$ and $\mathcal{N}$.

Moreover, if $(k-1)^{2}=(r-1)$

$$
R_{22}=\frac{1}{r-1} \iota\left(S-(k-1-r) I_{22}-\frac{1}{k-1} J_{22}\right), S_{22}=0
$$

If not, $R_{22}$ y $S_{22}$ are linearly independent and orthogonal.

Then $\mathcal{P}=\left\langle P_{11}\right\rangle, \mathcal{R}=\left\langle R_{22}\right\rangle, \mathcal{S}=\left\langle S_{22}\right\rangle$ are ideals of $\mathcal{T}$, orthogonal among them and orthogonal to $\mathcal{M}$ and to $\mathcal{N}$.

We get directly the following:

Theorem 4.5. Let $\mathcal{M}, \mathcal{N}, \mathcal{P}, \mathcal{R}, \mathcal{S} \subseteq \mathcal{T}$ be the simple ideals described above.

Then, the $\mathcal{T}$-algebra of a $G Q(k-1, r-1)$ has the following decomposition as a direct sum of orthogonal simple ideals:

$$
\begin{aligned}
\mathcal{T} & =\mathcal{M} \oplus \mathcal{N} \oplus \mathcal{P} \oplus \mathcal{R} \oplus \mathcal{S} \\
& \simeq \operatorname{End}\left(\mathbb{C}^{3}\right) \oplus \operatorname{End}\left(\mathbb{C}^{2}\right) \oplus \operatorname{End}\left(\mathbb{C}^{1}\right) \oplus \operatorname{End}\left(\mathbb{C}^{1}\right) \oplus \operatorname{End}\left(\mathbb{C}^{1}\right) \\
& \Longleftrightarrow(k-1)^{2} \neq r-1 \\
& =\mathcal{M} \oplus \mathcal{N} \oplus \mathcal{P} \oplus \mathcal{R} \\
& \simeq \operatorname{End}\left(\mathbb{C}^{3}\right) \oplus \operatorname{End}\left(\mathbb{C}^{2}\right) \oplus \operatorname{End}\left(\mathbb{C}^{1}\right) \oplus \operatorname{End}\left(\mathbb{C}^{1}\right) \\
& \Longleftrightarrow(k-1)^{2}=r-1
\end{aligned}
$$

Proof. It follows straightforward from Propositions 4.2, 4.3 and 4.4.

\section{Decomposition of $\mathbb{C}^{X}$ into irreducible $\mathcal{T}$-submodules}

In this section we consider the action of the $\mathcal{T}$-algebra

$$
\mathcal{T} \times \mathbb{C}^{X} \longrightarrow \mathbb{C}^{X}
$$

( $X$ is the set of vertices of the generalized quadrangle).

We have that

$$
\mathcal{T} \mathbb{C}^{X} \subseteq \mathbb{C}^{X}
$$

and since $I \in \mathcal{T}$ it holds

$$
\mathcal{T} \mathbb{C}^{X}=\mathbb{C}^{X}
$$

In the following we give a decomposition of $\mathbb{C}^{X}$ into irreducible left $\mathcal{T}$-submodules. 


\section{$5.1 \quad$ Isotypic left $\mathcal{T}$-submodules}

Let $\mathcal{T}=\mathcal{M} \oplus \mathcal{N} \oplus \mathcal{P} \oplus \mathcal{R} \oplus \mathcal{S}$

be the decomposition of Theorem 4.5. We can associate to each simple ideal a left $\mathcal{T}$-submodule in the following way:

$$
\begin{array}{ccc}
\{\text { simple ideals of } \mathcal{T}\}: & \rightarrow & \left\{\text { left } \mathcal{T} \text {-submodules of } \mathbb{C}^{X}\right\} \\
\mathcal{Z} & \rightarrow & \mathcal{Z} \mathbb{C}^{X}
\end{array}
$$

They are indeed left $\mathcal{T}$-submodules since by the orthogonality of the simple ideals we have

$$
\mathcal{T} \mathcal{Z} \mathbb{C}^{X} \subseteq \mathcal{Z} \mathbb{C}^{X} \text { for any simple ideal } \mathcal{Z} \in\{\mathcal{M}, \mathcal{N}, \mathcal{P}, \mathcal{R}, \mathcal{S}\}
$$

We call them isotypic $\mathcal{T}$-submodules.

Then the decomposition of $\mathbb{C}^{X}$ is :

$$
\begin{aligned}
\mathbb{C}^{X} & =\mathcal{M} \mathbb{C}^{X} \oplus \mathcal{N} \mathbb{C}^{X} \oplus \mathcal{P} \mathbb{C}^{X} \oplus \mathcal{R} \mathbb{C}^{X} \oplus \mathcal{S} \mathbb{C}^{X} \\
\mathcal{S} \mathbb{C}^{X} & =0 \Longleftrightarrow(k-1)^{2}=r-1
\end{aligned}
$$

\section{$5.2 \quad$ Irreducible left $\mathcal{T}$-submodules}

In this section we decompose each of the left isotypic $\mathcal{T}$-submodules into irreducible left $\mathcal{T}$ submodules.

To give the needed definitions we use as a guide the simple ideal $\mathcal{N}=\left\{N_{11}, N_{12}, N_{21}, N_{22}\right\}$ associated to the left isotypic $\mathcal{T}$-submodule $\mathcal{N} \mathbb{C}^{X}$.

The matrices of the basis satisfy

$$
N_{i j} N_{k l}=\delta_{j k} N_{i l} \quad i, j, k, l=1,2
$$

In particular, $\left\{N_{i i}\right\}_{i=1,2}$ are idempotents and they have a (not unique) decomposition as a sum of $r k\left(N_{i i}\right)$ projectors of rank one.(Here $r k(A)$ denote rank of $A$.)

That is, there exist

$$
\begin{aligned}
\left\{N_{11}^{(j)}\right\}_{j=1}^{r k\left(N_{11}\right)} & , \quad\left\{N_{22}^{(l)}\right\}_{l=1}^{r k\left(N_{22}\right)} \text { one-rank projectors such that } \\
N_{11}=\sum_{j=1}^{r k\left(N_{11}\right)} N_{11}^{(j)} & , \quad N_{22}=\sum_{l=1}^{r k\left(N_{22}\right)} N_{22}^{(l)} \text { which satisfy } \\
N_{i i}^{(j)} N_{i i}^{(k)}= & \delta_{j k} N_{i i}^{(j)} \text { for } i=1,2
\end{aligned}
$$


Remark 5.1. By equation (11) we have for example,

$$
\begin{aligned}
& N_{21}=N_{21} N_{11} \quad \text { then } \\
& N_{21}=\sum_{j=1}^{r k\left(N_{11}\right)} N_{21} N_{11}^{(j)}
\end{aligned}
$$

The remark carries out to define the following subspaces of $\mathcal{N} \mathbb{C}^{X}$

Definition 5.2. For $i=1, \ldots, r k\left(N_{11}\right)$

$$
W_{N_{11}^{(i)}}:=\left\{N_{11}^{(i)} v+\left(N_{21} N_{11}^{(i)}\right) w \quad v, w \in \mathbb{C}^{X} ;\right\}
$$

Then we have:

\section{Proposition 5.3.}

For $i=1, \ldots, r g\left(N_{11}\right) ; W_{N_{11}^{(i)}}$ is an irreducible left $\mathcal{T}$-submodule of dimension 2 and

$$
W_{N_{11}^{(i)}} \simeq W_{N_{11}^{(j)}}
$$

Proof. Equation (9) and the fact that mutually different ideals are orthogonal implies that $W_{N_{11}^{(i)}} \subseteq$ $\mathcal{N} \mathbb{C}^{X}$ and that $W_{N_{11}^{(i)}}$ is a left $\mathcal{T}$-submodule. $W_{N_{11}^{(i)}}$ is two dimensional since $N_{11}^{(i)}$ is a one-rank projector $\forall i=1, \ldots, r k\left(N_{11}\right)$.

Therefore given $\left\{e_{j}\right\}_{j=1}^{|X|}$ the canonical basis of $\mathbb{C}^{X}$, the subspace $\left\langle\left\{N_{11}^{(i)} e_{j}\right\}_{j=1}^{|X|}\right\rangle$ has dimension one as well has $\left\langle\left\{N_{21} N_{11}^{(i)} e_{j}\right\}_{j=1}^{|X|}\right\rangle$, which implies that $W_{N_{11}^{(i)}}$ has dimension two.

It is irreducible since if we consider a one dimensional subspace, it should be of the form

$$
\left\{\left(\alpha N_{11}^{(i)}+\beta N_{21} N_{11}^{(i)}\right) v ; v \in \mathbb{C}^{X}\right\}
$$

but the following actions of $\mathcal{T}$ would imply

$$
\begin{aligned}
N_{11}\left(\alpha N_{11}^{(i)}+\beta N_{21} N_{11}^{(i)}\right) \mathbb{C}^{X} & \subseteq \alpha N_{11}^{(i)} \mathbb{C}^{X} \Rightarrow \beta=0 \\
N_{22}\left(\alpha N_{11}^{(i)}+\beta N_{21} N_{11}^{(i)}\right) \mathbb{C}^{X} & \subseteq \beta N_{21} N_{11}^{(i)} \Rightarrow \alpha=0
\end{aligned}
$$

(which is a contradiction since it was a one dimensional subspace.)

It is easy to check that $W_{N_{11}^{(i)}} \simeq W_{N_{11}^{(j)}}$ considering the isomorphism:

$$
\begin{aligned}
\sigma_{\mathcal{N}}:\left(N_{11}^{(i)}+N_{21} N_{11}^{(i)}\right) \mathbb{C}^{X} & \longrightarrow\left(N_{11}^{(j)}+N_{21} N_{11}^{(j)}\right) \mathbb{C}^{X} \\
N_{11}^{(i)} v+N_{21} N_{11}^{(i)} w & \longrightarrow N_{11}^{(j)} v+N_{21} N_{11}^{(j)} w
\end{aligned}
$$

which preserve the action of $\mathcal{T}$.

\section{Proposition 5.4.}

$$
\mathcal{N} \mathbb{C}^{X}=\bigoplus_{j=1}^{r k\left(N_{11}\right)} W_{N_{11}^{(j)}}
$$


Proof. We have that $\sum_{j=1}^{r k\left(N_{11}\right)} W_{N_{11}^{(j)}} \subseteq \mathcal{N} \mathbb{C}^{X}$.

Conversely,

$$
\begin{aligned}
N_{11} \mathbb{C}^{X} & \subseteq \sum_{j=1}^{r k\left(N_{11}\right)} W_{N_{11}^{(j)}} \text { since by equation }(13) \\
N_{11} \mathbb{C}^{X} & =\left(\sum_{j=1}^{r k\left(N_{11}\right)} N_{11}^{(j)}\right) \mathbb{C}^{X} \subseteq \sum_{j=1}^{r k\left(N_{11}\right)} W_{N_{11}^{(j)}} . \text { Also } \\
N_{21} \mathbb{C}^{X} & \subseteq \sum_{j=1}^{r k\left(N_{11}\right)} W_{N_{11}^{(j)}}, \text { since } \\
N_{21} \mathbb{C}^{X}=N_{21} N_{11} \mathbb{C}^{X} & =N_{21}\left(\sum_{j=1}^{r k\left(N_{11}\right)} N_{11}^{(i)}\right) \mathbb{C}^{X} \\
& =\left(\sum_{j=1}^{r k\left(N_{11}\right)} N_{21} N_{11}^{(i)}\right) \mathbb{C}^{X} \\
& =\sum_{j=1}^{r k\left(N_{11}\right)}\left(N_{21} N_{11}^{(i)}\right) \mathbb{C}^{X}
\end{aligned}
$$

But we also have $N_{12} \mathbb{C}^{X} \subseteq \sum_{j=1}^{r k\left(N_{11}\right)} W_{N_{11}^{(j)}}$, since by equation (9)

$$
\begin{aligned}
N_{12} \mathbb{C}^{X} & =N_{12} \mathcal{N} \mathbb{C}^{X} \text { by equation (11) } \\
& =N_{11} \mathcal{N} \mathbb{C}^{X} \\
& =N_{11} \mathbb{C}^{X}
\end{aligned}
$$

$$
\text { Analogously } \begin{aligned}
N_{22} \mathbb{C}^{X} & =N_{22} \mathcal{N} \mathbb{C}^{X} \\
& =N_{21} \mathcal{N} \mathbb{C}^{X} \\
& =N_{21} \mathbb{C}^{X} .
\end{aligned}
$$

which implies $\sum_{j} W_{N_{11}^{(j)}} \supseteq \mathcal{N} \mathbb{C}^{X}$ and therefore the equality holds.

We will prove that it is a direct sum by comparing

$$
\operatorname{dim}\left(\sum_{j=1}^{r k\left(N_{11}\right)} W_{N_{11}^{(j)}}\right) \text { with } \operatorname{dim} \mathcal{N} \mathbb{C}^{X}
$$

We have $r k\left(N_{11}\right)$ 2-dimensional subspaces. By equation (14) and by definition of $W_{N_{11}^{(j)}}$ given in 5.2; it follows that

$$
\sum_{j=1}^{r k\left(N_{11}\right)} \operatorname{dim} W_{N_{11}^{(j)}}=2 r k\left(N_{11}\right)=2 \operatorname{tr}\left(N_{11}\right)=2 r(k-2) .
$$


On the other hand, we obtain the dimension of $\mathcal{N} \mathbb{C}^{X}$, computing the rank of the projection

$$
\begin{aligned}
& N \quad: \quad \mathbb{C}^{X} \rightarrow \mathcal{N} \mathbb{C}^{X} \\
& N=N_{11}+N_{22} \text { which has the form } \\
& =\left(\begin{array}{ccc}
0 & 0 & 0 \\
0 & \frac{\left((k-2) I_{11}-P\right)}{k-1} & 0 \\
0 & 0 & \frac{\left((k-1) Q^{t} Q-r J_{22}\right)}{(k-1)^{2}(r-1)}
\end{array}\right) .
\end{aligned}
$$

It is easy to check that

$$
\begin{aligned}
r k(N) & =\operatorname{tr}(N) \\
& =\operatorname{tr}\left(\frac{\left((k-2) I_{11}-P\right)}{k-1}\right)+\operatorname{tr}\left(\frac{\left((k-1) Q^{t} Q-r J_{22}\right)}{(k-1)^{2}(r-1)}\right) \\
\text { by Lemmas } 3.3 \text { and } 3.5 & =\frac{k-2}{k-1}\left|\Omega_{1}\right|+\frac{(k-1) r-r}{(k-1)^{2}(r-1)}\left|\Omega_{2}\right| \\
& =\frac{k-2}{k-1} r(k-1)+(k-2) r \\
& =2 r(k-2)
\end{aligned}
$$

Analogously we can decompose the other isotypic left $\mathcal{T}$-submodules. Considering the matrices $M_{i j}, P_{11}, R_{22}, S_{22}$ we define (the same way as for $W_{N_{11}^{(i)}}$ ),

\section{Definition 5.5.}

$$
\begin{aligned}
& W_{M_{00}}:=\left\{M_{00} u+M_{10} M_{00} v+M_{20} M_{00} w \quad u, v, w \in \mathbb{C}^{X}\right\} \\
& W_{P_{11}^{(i)}}:=\left\{P_{11}^{(i)} u \quad u \in \mathbb{C}^{X}, i=1, \ldots, r k\left(P_{11}\right)\right\} \\
& W_{R_{22}^{(i)}}:=\left\{R_{22}^{(i)} u \quad u \in \mathbb{C}^{X}, i=1, \ldots, r k\left(R_{22}\right)\right\} \\
& W_{S_{22}^{(i)}}:=\left\{S_{22}^{(i)} u \quad u \in \mathbb{C}^{X}, i=1, \ldots, r k\left(S_{22}\right)\right\}
\end{aligned}
$$

Then we have the following

\section{Theorem 5.6.}

$$
\mathbb{C}^{X}=W_{M_{00}} \oplus_{j=1}^{r(k-2)} W_{N_{11}^{(j)}} \oplus_{j=1}^{r-1} W_{P_{11}^{(j)}} \oplus_{j=1}^{d_{R}} W_{R_{22}^{(j)}} \oplus_{j=1}^{d_{S}} W_{S_{22}^{(j)}}
$$

and

$$
\begin{aligned}
\mathcal{M} \mathbb{C}^{X} & =W_{M_{00}} \text { where } W_{M_{00}} \text { is an irreducible left } \mathcal{T} \text {-module of dimension } 3 \\
\mathcal{P} \mathbb{C}^{X} & =\oplus_{j=1}^{r-1} W_{P_{11}^{(j)}} \text { where } W_{P_{11}^{j}} \text { are irreducible left } \mathcal{T} \text {-modules of dimension } 1 \\
\mathcal{R} \mathbb{C}^{X} & =\oplus_{j=1}^{d_{R}} W_{R_{22}^{(j)}} \text { where } W_{R_{22}^{(j)}} \text { are irreducible left } \mathcal{T} \text {-modules of dimension } 1 \\
\mathcal{S} \mathbb{C}^{X} & =\oplus_{j=1}^{d_{S}} W_{S_{22}^{(j)}} \text { where } W_{S_{22}^{(j)}} \text { are irreducible left } \mathcal{T} \text {-modules of dimension } 1
\end{aligned}
$$


where

$$
d_{R}=\frac{r(r-2)(k-1)^{2}}{(k-2+r)}, d_{S}=\frac{(r-1)(k-2)\left((k-1)^{2}-(r-1)\right)}{(k-2+r)}
$$

and

$$
r k\left(M_{00}\right)=1, r k\left(P_{11}\right)=(r-1), r k\left(R_{22}\right)=d_{R}, r k\left(S_{22}\right)=d_{S}
$$

Proof. The proof is analogous to the one given for the decomposition of $\mathcal{N} \mathbb{C}^{X}$.

The number of irreducible left $\mathcal{T}$-submodules that appear on each decomposition depends on the rank of the projections to corresponding isotypic left $\mathcal{T}$-submodule:

$$
\begin{aligned}
M & : \mathbb{C}^{X} \rightarrow \mathcal{M} \mathbb{C}^{X} \\
M= & M_{00}+M_{11}+M_{22} \\
= & \left(\begin{array}{ccc}
1 & 0 & 0 \\
0 & \frac{J_{11}}{\sqrt{\left|\Omega_{1}\right|\left|\Omega_{1}\right|}} & 0 \\
0 & 0 & \frac{J_{22}}{\sqrt{\left|\Omega_{2}\right|\left|\Omega_{2}\right|}}
\end{array}\right) . \\
P \quad: & \mathbb{C}^{X} \rightarrow \mathcal{P} \mathbb{C}^{X} \quad P:=P_{11} \\
R \quad: & \mathbb{C}^{X} \rightarrow \mathcal{R} \mathbb{C}^{X} \quad R:=R_{22} \\
S: & \mathbb{C}^{X} \rightarrow \mathcal{S} \mathbb{C}^{X} \quad S:=S_{22}
\end{aligned}
$$

From the definition of such matrices, and computing its trace, we get the corresponding ranks.

\section{Corollary 5.7.}

$$
k(1+(k-1)(r-1))=3+2 r(k-2)+r-1+\frac{r(r-2)(k-1)^{2}}{(k-2+r)}+\frac{(r-1)(k-2)\left((k-1)^{2}-(r-1)\right)}{(k-2+r)}
$$

Proof. One can get the equation by computing the dimensions of the decomposition given in Theorem 5.6 .

Remark 5.8. In subsections $5.1,5.2$ we can exchange "left" by "right" considering the action of the $\mathcal{T}$-algebra

$$
\mathbb{C}^{X} \times \mathcal{T} \longrightarrow \mathbb{C}^{X}
$$

that gives

$$
\mathbb{C}^{X} \mathcal{T}=\mathbb{C}^{X}
$$

Acknowledgement. This work was finished during a visiting research position at the Department of Mathematics of UFPE, Recife-Brasil (september-november 2008) supported by FACEPE and CIEM-CONICET. We would like to thank Professors Manoel Lemos and Fernando Souza for the invitation. 
Some topics of this paper were developed in the regular seminar of the research group of Estruturas Discretas of such a Department.

We also wish to thank Paul Terwilliger for introducing us into the subject.

$$
\text { Received: November 2008. Revised: February } 2009 .
$$

\section{References}

[1] R.C. Bose, Strongly regular graphs, partial geometries and partially balanced designs. Pac. J. Math. 13, 389-419 (1963)

[2] A. E. Brouwer, A. M. Cohen and A. Neumaier, Distance-regular graphs. Ergebnisse der Mathematik und ihrer Grenzgebiete. 3. Folge, 18. Berlin etc.: Springer-Verlag. xvii, 495 p. (1989).

[3] E. Bannai and T. Ito, Algebraic Combinatorics I:Association Schemes. Benjamin Cummings, London, 1984.

[4] E. Bannai And A. Munemasa, The Terwilliger algebras of group association schemes. Kyushu J. Math. 49, No.1, 93-102 (1995).

[5] J. M. Balmaceda And M. Oura, The Terwilliger algebras of the group association schemes of $S_{5}$ and $A_{5}$. Kyushu J. Math. 48, No.2, 221-231 (1994).

[6] J. S. Caughman IV, The Terwilliger algebras of bipartite $P$ - and $Q$-polynomial schemes. Discrete Math. 196, No.1-3, 65-95 (1999).

[7] J. S. Caughman and N. WolfF, The Terwilliger algebra of a distance-regular graph that supports a spin model. J. Algebr. Comb. 21, No. 3, 289-310 (2005).

[8] J. T. Go, The Terwilliger algebra of the hypercube. Eur. J. Comb. 23, No.4, 399-429 (2002).

[9] F. Levstein and C. Maldonado, The Terwilliger algebra of the Johnson schemes. Discrete Math. 307, No. 13, 1621-1635 (2007).

[10] F. Levstein, C. Maldonado and D. Penazzi, The Terwilliger algebra of a Hamming scheme $H(d, q)$. Eur. J. Comb. 27, No. 1, 1-10 (2006).

[11] J. H. van Lint and R. Wilson, A course in Combinatorics, Cambridge U.P.(1992)

[12] S. E. Payne and J. A. Thas, Finite generalized quadrangles, Pitman Advanced Publishing (1984).

[13] Paul Terwilliger, The subconstituent algebra of an association scheme. I. J. Algebr. Comb. 1, No.4, 363-388 (1992).

[14] Paul Terwilliger, The subconstituent algebra of an association scheme. II. J. Algebr. Comb. 2, No.1, 73-103 (1993). 
[15] Paul Terwilliger, The subconstituent algebra of an association scheme. III. J. Algebr. Comb. 2, No.2, 177-210 (1993).

[16] J.A. ThAs, Combinatorial characterizations of generalized quadrangles with parameters $s=q$ and $t=q^{2}$. Geom. Dedicata 7, 223-232 (1978).

[17] M. Tomiyama And N. Yamazaki, The subconstituent algebra of a strongly regular graph. Kyushu J. Math. 48, No.2, 323-334 (1994). 